\title{
Host Plant Preference of Sucking Pest to Different Tomato Genotypes under West Bengal Conditions
}

\author{
Prahlad Sarkar $^{1^{*}}$, Satyajit Hembram ${ }^{2}$ and Siddikul Islam ${ }^{3}$ \\ ${ }^{1}$ All India Network Project on Jute and Allied Fibres, Uttar Banga Krishi Viswavidyalaya, \\ Pundibari, Cooch Behar, West Bengal, India \\ ${ }^{2}$ Regional Research Station, Terai Zone, Uttar Banga Krishi Viswavidyalaya, Pundibari, \\ Cooch Behar, West Bengal, India \\ ${ }^{3}$ Dakshin Dinajpur KVK, Uttar Banga Krishi Viswavidyalaya, Majhian, West Bengal, India \\ *Corresponding author
}

\section{A B S T R A C T}

A field study was conducted to screen out six important tomato genotypes for resistance/ tolerance against important sucking pests and their natural enemies under West Bengal conditions. Aphid (Aphis gossypii Glover) and whitefly (Bemisia tabaci Genn.) were first

Keywords

Tomato genotypes,

Sucking pests,

Natural enemies,

Resistance

\section{Article Info}

Accepted:

26 October 2018

Available Online:

10 November 2018 appeared on the crop in third week of January and second week of February respectively. The peak populations of both the pest were reached in the third to fourth week of February. The maximum $(r=-0.027$ and -0.210$)$ and minimum temperatures $(r=-0.138$ and -0.283 ) and minimum relative humidity $(\mathrm{r}=-0.191$ and -0.031$)$ were found to exert unfavorable influence on population development of whitefly and aphid species and showed negative correlation whereas maximum relative humidity $(\mathrm{r}=0.225$ and 0.428$)$ and sunshine hour $(r=0.547$ and 0.387$)$ favoured the population build up. Results showed that none of tested tomato genotypes were found either as tolerant or resistant against aphid and whitefly. In terms of relative susceptibility against aphid and whitefly, the tomato genotypes could be arranged in the following sequence (higher to lower): NS 501>Priya>Roja> Romeo $>$ Rubi $>$ Patherkuchi and NS 501> Romeo $>$ Rubi $>$ Priya $>$ Roja $>$ Patherkuchi respectively. Here Patherkuchi was found less susceptible for both the pest aphid (16.05/leaf) and whitefly (0.20/leaf) as well as found to be immune $(0.00 \%)$ against ToLCV disease incidence. Remaining other genotypes were moderately susceptible (Ruby, Roja cherry, Romeo and Priya) to highly susceptible NS501 (20.77/leaf and 0.40/leaf).

\section{Introduction}

Tomato (Lycopersicon esculentum Mill.) is one of the important vegetable crops belongs to nightshade family (along with aubergines, peppers and chillies), that have a high appealing for farmers because of its greater yield and comparatively short growing period
(Naika et al., 2005; Alam et al., 2007). Tomatoes originated in western South America, crossed the Atlantic to Spain with the conquistadors in the 16th century, but only finally caught on in northern Europe in the 19th century (Acquaah, 2002). Total area under tomato crop in the country is about 8.8 million hectares with a total yield of about 
182.27 lakhtonnes and an average yield of 20.70tonnes/ha (NHPD, 2012).

A tomato is the edible, often red fruit from the plant Solanum lycopersicum. The crop is also known as industrial crop because of its taste, nutritive values and outstanding processing qualities. Tomato is one of the likable crops among the vegetables because of its diverse ways of consumption, including raw, as an ingredient in many dishes, sauces, salads, and drinks. The fruit is rich in lycopene, which may have beneficial health effects. Tomato consumption has been associated with decreased risk of breast cancer (Zhang et al., 2009), head and neck cancers (Freedman et al., 2008) and might be beneficial for reducing cardiovascular risk associated with type 2 diabetes (Shidfar et al., 2011).

However, like all other vegetable crops, tomato also faces some production hurdles due to unfavorable temperature, moisture-stress, cracking, pollination, pests etc. but the problem posed by pests is very critical. At times, it may become very critical and if emergency measures are not contemplated, the entire yield may have to be compromised.

Tomatoes are subject to attack by quite a large spectrum of insect pests along with aphid (Aphis gossypii Glover) and whitefly (Bemisiatabaci Genn.) from the time of seed emergence to harvest. To avoid the crop loss, onus primarily fell on the exploitation of chemical method (Anonnymous, 2011; Raddy, 2010; Sukla and Prabhakar, 1987; Musuna, 1983). But it has got a large array of nontarget impacts and that makes it unfit to be adopted as a steady option. To obtain an acceptable yield level and cost-benefit ratio, the pest problem needs to be effectively addressed. Present-day -pest-management emphasizes on a holistic approach that cares for the plant, pest, beneficial organisms as well as the environment. Hence, the rationale should emphasize on the principle of "live and let live". It allows sustainability and stability to the entire crop ecosystem and eventually ensures good yields.

Resistance technology occupies an important place in the present day rational pest management strategies. A genotype exhibits tolerance or resistance by any of the three basic resistance mechanisms: preference-non preference, antibiosis and tolerance. Keeping this option of utilizing resistance technology in mind some tomato germplasms were screened for their relative tolerance/ susceptibility to the major sucking pest of the region.

\section{Materials and Methods}

The experiment was carried out at A-B Block Research Farm, B.C.K.V., Kalyani, Nadia, West Bengal. The studies were conducted during January 2013 to April 2013. Standard agronomic practices were followed during different growth stages of crop. The entire field experiment was conducted under unsprayed condition.

The experimental site is situated at $22.980 \mathrm{~N}$ latitude and $88.480 \mathrm{E}$ longitude and $9.75 \mathrm{~m}$ above mean sea level. During the study period, maximum temperature ranged between $24.44-36.76^{\circ} \mathrm{C}$ and minimum temperature varied from $7.59-23.24^{\circ} \mathrm{C}$. Generally winter is very short and mild in this region. The area comes under gangetic new alluvial zone plains. The soil is sandy loam type and slightly acidic $(\mathrm{pH}$ 6.5) in nature, low in organic carbon $(0.54 \%)$ and available nitrogen $(0.69 \%)$. The available phosphorus (30.0 $\mathrm{kg} / \mathrm{ha})$ and potassium $(240 \mathrm{~kg} / \mathrm{ha})$ contents are medium.

Total six genotypes of tomato including established varieties as well as local types were tested for their resistance/tolerance to 
important insect pests. Existing natural enemies in the tomato ecosystem were also included in the study. The morphological characters of the genotypes are listed in table1. The experiment was conducted in randomized block design with four replications and six tomato genotypes as the treatments.

\section{Raising the crop}

The seeds of all germolasms were sown in nursery bed on 1st January following standard norms and 30 day-old-seedlings were transplanted at a spacing of $75 \mathrm{~cm} \times 75 \mathrm{~cm}$ in $3 \mathrm{~m} \times 3 \mathrm{~m}$ plots. Fertilizers were applied @ 120:50:50 (N: P: K). Nitrogen was applied in 3 split doses, first one at the time of transplanting, second one at 30 days after transplanting and last one 30 days after first top dressing. Full dose of $\mathrm{P}$ and $\mathrm{K}$ was given at the time of transplanting.

\section{Method of observation}

To assess the insect infestation, data were collected from randomly selected plants at an interval of seven days starting from 27January 2013 to 30 April 2013.Four plants were randomly selected from each replication for observations on aphid and whitefly.

The number of aphid and whitefly (nymph and adult) present on the plants were counted at 7 day-interval from randomly selected 3 leaves (1 tender, 1 moderately old, 1 old) from three tiers (upper, middle, lower) of the crop canopy of each of four plants. Total numbers of the natural enemies were also counted from randomly selected 4 plants/replication. The natural enemies were -3 species of spiders, 4 species of coccinellids and 1 species of hover fly.

Genotypes were designated as less susceptible (LS), moderately susceptible (MS), highly susceptible (HS) and tolerant /resistant (T/R) types depending on their reaction to insect infestation.

To assess the ToLCV causing disease in tomato, total number of diseased and healthy plants per plot was recorded at 60 days after transplanting by counting leaf curled plants.

Disease reaction on the different genotypes was categories by following Singh (2014) and the percent disease incidence was calculated through using the following formula.

Leaf curl infected plants (\%)= Leaf curled (infected) plants/plot

Total number of plants/plot $\quad$ X100

\section{Results and Discussion}

The population of sucking pest including aphid and whitefly were recorded during the investigation presented below.

\section{Tomato germplasms and whitefly}

The white fly impacts the tomato plants in three ways: direct injury due to puncture and sap removal, sugary secretion causing sooty mould growth which eventually interferes with photosynthesis and vectoring of leaf curl and yellow vein mosaic viruses.

The results (Table 2) showed that the pest first appeared on the crop in second week of February and the peak was reached in the fourth week of February.

The maximum $(\mathrm{r}=-0.027)$ and minimum temperatures $(\mathrm{r}=-0.138)$ and minimum relative humidity $(\mathrm{r}=-0.191)$ were found to exert unfavorable influence on population development of the pest species and showed negative correlation whereas maximum relative humidity ( $\mathrm{r}=0.225)$ and sunshine hour $(r=0.547)$ favoured the population build up. 
The germplasms showed differential response to white fly infestation. The highest number of the fly was recorded in germplasm NS501 (0.40) followed by Romeo (0.38). Patherkuchi had a mean 0.20 white fly/leaf while Roja cherry (0.30) and Priya (0.30) had a marginally higher mean population. In terms of relative tolerance, the tomato germplasms can be arranged in the following sequence (higher to lower): Patherkuchi>Roja> Priya $>$ Rubi> Romeo > NS 501.

Results as indicated in the Table 3 revealed that among the six genotypes, local variety Patherkuchi was found to be immune $(0.00 \%)$ without any symptoms of ToLCV disease incidence. Roja and Priya exhibited ToLCV infection ranging $\leq 5.00 \%$ and showed resistance reaction to ToLCV. Moderately resistant reaction was found in NS 501 (8.33\%) and Romeo (6.25\%). One variety Ruby $(10.42 \%)$ was observed moderately susceptible to ToLCV infection.

\section{Tomato germplasms and aphid}

Nymphs and adults of aphids usually remain on lower surface of leaves, growing apical primordia and succulent stems. Infested leaves curled downward, appear yellowish and growth is greatly retarded.

Infested young shoots may dry up. Infestation in early stage cause stunting of the plants.

The pest was first appeared on the crop in third week of January (Table 4). In the most of the tomato genotypes, the pest population reached its critical level during third to fourth week of February.

The maximum temperature $(\mathrm{r}=-0.210)$, minimum temperature $(\mathrm{r}=-0.283)$ and minimum relative humidity $(\mathrm{r}=-0.031)$ showed negative correlation with the pest species while maximum relative humidity $(\mathrm{r}=0.428)$ and sunshine hour $(\mathrm{r}=0.387)$ showed positive correlation.

The lowest aphid population was recorded on Patherkuchi (16.05) followed by Ruby (16.69). NS501 (20.77) showed maximum Aphid population followed by BSS 908 Priya (19.29) and the order of relative tolerance can be arranged as (high to low): Patherkuchi>Rubi $>$ Romeo>Roja $>$ Priya $>$ NS 501.

Table.1 Gross morphological characters of the tomato genotypes

\begin{tabular}{|c|c|c|c|c|c|c|c|}
\hline Variety & $\begin{array}{c}\text { Plant } \\
\text { height } \\
\text { (cm) }\end{array}$ & $\begin{array}{c}\text { Plant } \\
\text { canopy } \\
(\mathbf{c m})\end{array}$ & $\begin{array}{c}\text { Mean } \\
\text { fruit } \\
\text { number }\end{array}$ & $\begin{array}{c}\text { Mean } \\
\text { fruit } \\
\text { weight } \\
(\mathbf{g})\end{array}$ & $\begin{array}{c}\text { Mean } \\
\text { fruit } \\
\text { diameter } \\
(\mathbf{c m})\end{array}$ & $\begin{array}{c}\text { Leaf } \\
\text { character } \\
\text { (glabrous or } \\
\text { hairy) }\end{array}$ & $\begin{array}{c}\text { Stem } \\
\text { character } \\
\text { (soft or } \\
\text { compact) }\end{array}$ \\
\hline $\begin{array}{c}\text { Patherkuch } \\
\text { i }\end{array}$ & 98.58 & 50.83 & 21.62 & 43.48 & 4.06 & glabrous & soft \\
\hline NS501 & 88.33 & 45.42 & 32.58 & 43.71 & 4.26 & hairy & compact \\
\hline Romeo & 87.83 & 45.83 & 38.22 & 41.00 & 3.92 & hairy & compact \\
\hline Ruby & 68.75 & 38.25 & 33.29 & 31.42 & 3.61 & glabrous & soft \\
\hline Roja & 78.75 & 36.25 & 70.58 & 5.65 & 2.14 & hairy & compact \\
\hline BSS908 & 79.68 & 40.67 & 32.22 & 34.80 & 3.69 & glabrous & soft \\
\hline Priya & & & & & & &
\end{tabular}


Table.2 Mean number of Bemisiatabaci Genn. per leaf ondifferent tomato genotypes

\begin{tabular}{|c|c|c|c|c|c|c|c|c|c|c|c|c|}
\hline \multirow[t]{2}{*}{ Variety } & \multicolumn{11}{|c|}{ Date of observation(at 7 days interval) } & \multirow[t]{2}{*}{ Mean } \\
\hline & $\begin{array}{c}27.1 .1 \\
3\end{array}$ & $\begin{array}{c}03.02 .1 \\
3\end{array}$ & $\begin{array}{c}10.02 .1 \\
3\end{array}$ & $\begin{array}{c}17.02 .1 \\
3\end{array}$ & $\begin{array}{c}24.02 .1 \\
3\end{array}$ & $\begin{array}{c}\text { 03.03.1 } \\
3\end{array}$ & $\begin{array}{c}10.03 .1 \\
3\end{array}$ & $\begin{array}{c}17.03 .1 \\
3\end{array}$ & $\begin{array}{c}24.03 .1 \\
3\end{array}$ & $\begin{array}{c}31.03 .1 \\
3\end{array}$ & $\begin{array}{c}07.04 .1 \\
3\end{array}$ & \\
\hline Patherkuchi & $\begin{array}{c}0.00 \\
(4.05)\end{array}$ & $\begin{array}{c}0.00 \\
(4.05)\end{array}$ & $\begin{array}{c}0.00 \\
(4.05)\end{array}$ & $\begin{array}{c}0.13 \\
(4.51)\end{array}$ & $\begin{array}{c}0.40 \\
(5.42)\end{array}$ & $\begin{array}{c}0.81 \\
(6.57)\end{array}$ & $\begin{array}{c}0.56 \\
(5.91)\end{array}$ & $\begin{array}{c}0.33 \\
(5.23)\end{array}$ & $\begin{array}{c}0.00 \\
(4.05)\end{array}$ & $\begin{array}{c}0.00 \\
(4.05)\end{array}$ & $\begin{array}{c}0.00 \\
(4.05)\end{array}$ & 0.20 \\
\hline NS501 & $\begin{array}{c}0.00 \\
(4.05)\end{array}$ & $\begin{array}{c}0.00 \\
(4.05)\end{array}$ & $\begin{array}{c}0.00 \\
(4.05)\end{array}$ & $\begin{array}{c}0.29 \\
(5.10)\end{array}$ & $\begin{array}{c}1.06 \\
(7.14)\end{array}$ & $\begin{array}{c}1.63 \\
(8.38)\end{array}$ & $\begin{array}{c}1.06 \\
(7.16)\end{array}$ & $\begin{array}{c}0.31 \\
(5.16)\end{array}$ & $\begin{array}{c}0.00 \\
(4.05)\end{array}$ & $\begin{array}{c}0.00 \\
(4.05)\end{array}$ & $\begin{array}{c}0.00 \\
(4.05)\end{array}$ & 0.40 \\
\hline Romeo & $\begin{array}{c}0.00 \\
(4.05)\end{array}$ & $\begin{array}{c}0.00 \\
(4.05)\end{array}$ & $\begin{array}{c}0.00 \\
(4.05)\end{array}$ & $\begin{array}{c}0.29 \\
(5.10)\end{array}$ & $\begin{array}{c}0.63 \\
(6.08)\end{array}$ & $\begin{array}{c}1.56 \\
(8.25)\end{array}$ & $\begin{array}{c}1.00 \\
(7.01)\end{array}$ & $\begin{array}{c}0.56 \\
(5.91)\end{array}$ & $\begin{array}{c}0.19 \\
(4.74)\end{array}$ & $\begin{array}{c}0.00 \\
(4.05)\end{array}$ & $\begin{array}{c}0.00 \\
(4.05)\end{array}$ & 0.38 \\
\hline Ruby & $\begin{array}{c}0.00 \\
(4.05)\end{array}$ & $\begin{array}{c}0.00 \\
(4.05)\end{array}$ & $\begin{array}{c}0.00 \\
(4.05)\end{array}$ & $\begin{array}{c}0.29 \\
(5.10)\end{array}$ & $\begin{array}{c}0.69 \\
(6.23)\end{array}$ & $\begin{array}{c}1.56 \\
(8.25)\end{array}$ & $\begin{array}{c}1.00 \\
(7.01)\end{array}$ & $\begin{array}{c}0.40 \\
(5.42)\end{array}$ & $\begin{array}{c}0.00 \\
(4.05)\end{array}$ & $\begin{array}{c}0.00 \\
(4.05)\end{array}$ & $\begin{array}{c}0.00 \\
(4.05)\end{array}$ & 0.36 \\
\hline $\begin{array}{c}\text { Roja } \\
\text { (Cherry) }\end{array}$ & $\begin{array}{c}0.00 \\
(4.05)\end{array}$ & $\begin{array}{c}0.00 \\
(4.05)\end{array}$ & $\begin{array}{c}0.00 \\
(4.05)\end{array}$ & $\begin{array}{c}0.29 \\
(5.10)\end{array}$ & $\begin{array}{c}0.69 \\
(6.25)\end{array}$ & $\begin{array}{c}1.38 \\
(7.87)\end{array}$ & $\begin{array}{c}0.63 \\
(6.08)\end{array}$ & $\begin{array}{c}0.27 \\
(5.03)\end{array}$ & $\begin{array}{c}0.00 \\
(4.05)\end{array}$ & $\begin{array}{c}0.00 \\
(4.05)\end{array}$ & $\begin{array}{c}0.00 \\
(4.05)\end{array}$ & 0.30 \\
\hline $\begin{array}{c}\text { BSS908 } \\
\text { (Priya) } \\
\end{array}$ & $\begin{array}{c}0.00 \\
(4.05)\end{array}$ & $\begin{array}{c}0.00 \\
(4.05)\end{array}$ & $\begin{array}{c}0.00 \\
(4.05)\end{array}$ & $\begin{array}{c}0.33 \\
(5.23)\end{array}$ & $\begin{array}{c}0.63 \\
(6.08)\end{array}$ & $\begin{array}{c}1.40 \\
(7.89)\end{array}$ & $\begin{array}{c}0.63 \\
(6.08)\end{array}$ & $\begin{array}{c}0.31 \\
(5.16)\end{array}$ & $\begin{array}{c}0.00 \\
(4.05)\end{array}$ & $\begin{array}{c}0.00 \\
(4.05)\end{array}$ & $\begin{array}{c}0.00 \\
(4.05)\end{array}$ & 0.30 \\
\hline S.Em \pm & 0.00 & 0.00 & 0.00 & 0.13 & 0.25 & 0.17 & 0.21 & 0.13 & 0.08 & 0.00 & 0.00 & \\
\hline $\mathrm{CD}(=0.05)$ & 0.00 & 0.00 & 0.00 & 0.39 & 0.74 & 0.51 & 0.63 & 0.40 & 0.25 & 0.00 & 0.00 & \\
\hline $\mathrm{CV}=$ & 0.00 & 0.00 & 0.00 & 5.80 & 8.89 & 4.77 & 7.18 & 5.61 & 4.47 & 0.00 & 0.00 & \\
\hline
\end{tabular}

Figure in parenthesis signifies the transformation of original value

Table.3 Reaction of tomato genotypes against ToLCV causing leaf curl disease

\begin{tabular}{|c|c|c|c|}
\hline S. No. & Genotype & Leaf curl incidence (\%) & Reaction \\
\hline 1. & Patherkuchi & 0.00 & Immune \\
\hline 2. & NS501 & 8.33 & Moderately resistant \\
\hline 3. & Romeo & 6.25 & Moderately resistant \\
\hline 4. & Ruby & 10.42 & Moderately susceptible \\
\hline 5. & Roja (Cherry) & 4.16 & Resistant \\
\hline 6. & BSS908 (Priya) & 2.08 & Resistant \\
\hline
\end{tabular}

Table.4 Aphis gossypii Glover population on tomato plants (mean number per three leaves)

\begin{tabular}{|c|c|c|c|c|c|c|c|c|c|c|c|c|}
\hline \multirow[t]{2}{*}{ Variety } & \multicolumn{11}{|c|}{ Date of observation(at 7 days interval) } & \multirow[t]{2}{*}{ Mean } \\
\hline & 27.1.13 & 03.02.13 & 10.02.13 & 17.02.13 & 24.02.13 & 03.03.13 & 10.03 .13 & 17.03.13 & 24.03.13 & 31.03.13 & 07.04.13 & \\
\hline Patherkuchi & $\begin{array}{c}4.06 \\
(12.32)\end{array}$ & $\begin{array}{c}5.50 \\
(14.16)\end{array}$ & $\begin{array}{c}7.38 \\
(16.29)\end{array}$ & $\begin{array}{c}28.56 \\
(32.60)\end{array}$ & $\begin{array}{c}35.44 \\
(36.83)\end{array}$ & $\begin{array}{c}44.19 \\
(41.94)\end{array}$ & $\begin{array}{c}27.31 \\
(31.80)\end{array}$ & $\begin{array}{c}19.00 \\
(26.14)\end{array}$ & $\begin{array}{c}5.06 \\
(13.63)\end{array}$ & $\begin{array}{c}0.00 \\
(4.05)\end{array}$ & $\begin{array}{c}0.00 \\
(4.05)\end{array}$ & 16.05 \\
\hline NS501 & $\begin{array}{c}5.06 \\
(13.62)\end{array}$ & $\begin{array}{c}6.50 \\
(15.34)\end{array}$ & $\begin{array}{c}7.50 \\
(16.42)\end{array}$ & $\begin{array}{c}34.19 \\
(36.03)\end{array}$ & $\begin{array}{c}49.81 \\
(45.18)\end{array}$ & $\begin{array}{c}58.31 \\
(50.08)\end{array}$ & $\begin{array}{c}36.63 \\
(37.49)\end{array}$ & $\begin{array}{c}25.44 \\
(30.61)\end{array}$ & $\begin{array}{c}5.00 \\
(13.55)\end{array}$ & $\begin{array}{c}0.00 \\
(4.05)\end{array}$ & $\begin{array}{c}0.00 \\
(4.05)\end{array}$ & 20.77 \\
\hline Romeo & $\begin{array}{c}4.31 \\
(12.64)\end{array}$ & $\begin{array}{c}6.31 \\
(15.02)\end{array}$ & $\begin{array}{c}8.19 \\
(17.07)\end{array}$ & $\begin{array}{c}30.69 \\
(33.93)\end{array}$ & $\begin{array}{c}38.56 \\
(38.62)\end{array}$ & $\begin{array}{c}45.94 \\
(42.95)\end{array}$ & $\begin{array}{c}27.19 \\
(31.72)\end{array}$ & $\begin{array}{c}21.88 \\
(28.21)\end{array}$ & $\begin{array}{c}4.63 \\
(13.07)\end{array}$ & $\begin{array}{c}0.00 \\
(4.05)\end{array}$ & $\begin{array}{c}0.00 \\
(4.05)\end{array}$ & 17.06 \\
\hline Ruby & $\begin{array}{c}6.75 \\
(15.61)\end{array}$ & $\begin{array}{c}7.63 \\
(16.56)\end{array}$ & $\begin{array}{c}10.38 \\
(19.24)\end{array}$ & $\begin{array}{c}29.38 \\
(33.11)\end{array}$ & $\begin{array}{c}38.00 \\
(38.34)\end{array}$ & $\begin{array}{c}58.13 \\
(50.11)\end{array}$ & $\begin{array}{c}27.75 \\
(32.09)\end{array}$ & $\begin{array}{c}24.00 \\
(29.66)\end{array}$ & $\begin{array}{c}4.44 \\
(12.80)\end{array}$ & $\begin{array}{c}0.56 \\
(5.69)\end{array}$ & $\begin{array}{c}0.00 \\
(4.05)\end{array}$ & 16.69 \\
\hline $\begin{array}{c}\text { Roja } \\
\text { (Cherry) }\end{array}$ & $\begin{array}{c}4.06 \\
(12.32)\end{array}$ & $\begin{array}{c}4.94 \\
(13.48)\end{array}$ & $\begin{array}{c}6.06 \\
(14.83)\end{array}$ & $\begin{array}{c}21.19 \\
(27.72)\end{array}$ & $\begin{array}{c}38.88 \\
(38.85)\end{array}$ & $\begin{array}{c}47.31 \\
(43.74)\end{array}$ & $\begin{array}{c}37.31 \\
(37.85)\end{array}$ & $\begin{array}{c}20.94 \\
(27.57)\end{array}$ & $\begin{array}{c}2.94 \\
(10.65)\end{array}$ & $\begin{array}{c}0.00 \\
4.05)\end{array}$ & $\begin{array}{c}0.00 \\
(4.05)\end{array}$ & 18.82 \\
\hline $\begin{array}{c}\text { BSS908 } \\
\text { (Priya) } \\
\end{array}$ & $\begin{array}{c}6.50 \\
(15.33)\end{array}$ & $\begin{array}{c}7.38 \\
(16.27)\end{array}$ & $\begin{array}{c}8.69 \\
(17.61)\end{array}$ & $\begin{array}{c}28.81 \\
(32.69)\end{array}$ & $\begin{array}{c}40.69 \\
(39.89)\end{array}$ & $\begin{array}{c}59.63 \\
(50.95)\end{array}$ & $\begin{array}{c}30.06 \\
(33.47)\end{array}$ & $\begin{array}{c}24.19 \\
(29.77)\end{array}$ & $\begin{array}{c}5.56 \\
(14.19)\end{array}$ & $\begin{array}{c}0.69 \\
(5.96)\end{array}$ & $\begin{array}{c}0.00 \\
(4.05)\end{array}$ & 19.29 \\
\hline S.Em \pm & 0.37 & 0.49 & 0.46 & 1.11 & 1.28 & 1.97 & 1.49 & 0.73 & 0.43 & 0.48 & 0.00 & \\
\hline $\mathrm{CD}(=0.05)$ & 1.10 & 1.49 & 1.39 & 3.35 & 3.87 & 5.92 & 4.49 & 2.20 & 1.30 & 1.44 & 0.00 & \\
\hline $\mathrm{CV}=$ & 5.98 & 7.29 & 6.11 & 7.60 & 7.25 & 9.42 & 9.78 & 5.70 & 7.46 & 23.01 & 0.00 & \\
\hline
\end{tabular}

Figure in parenthesis signifies the transformation of original value 
Table.5 Mixed population of the natural enemies (spiders, coccinellids and hover fly) in tomato ecosystem

\begin{tabular}{|c|c|c|c|c|c|c|c|c|c|c|c|c|}
\hline \multirow[t]{2}{*}{ Variety } & \multicolumn{11}{|c|}{ Date of observation(at 7 days interval) } & \multirow[t]{2}{*}{ Mean } \\
\hline & 27.1.13 & 03.02.13 & 10.02 .13 & 17.02.13 & 24.02.13 & 03.03.13 & 10.03 .13 & 17.03.13 & 24.03.13 & 31.03.13 & 07.04.13 & \\
\hline Patherkuchi & $\begin{array}{c}0.00 \\
(4.05)\end{array}$ & $\begin{array}{c}0.00 \\
(4.05)\end{array}$ & $\begin{array}{c}0.00 \\
(4.05)\end{array}$ & $\begin{array}{c}0.19 \\
(4.74)\end{array}$ & $\begin{array}{c}0.31 \\
(5.16)\end{array}$ & $\begin{array}{c}0.63 \\
(6.08)\end{array}$ & $\begin{array}{c}1.19 \\
(7.45)\end{array}$ & $\begin{array}{c}0.88 \\
(6.71)\end{array}$ & $\begin{array}{c}0.63 \\
(6.08)\end{array}$ & $\begin{array}{c}0.50 \\
(5.74)\end{array}$ & $\begin{array}{c}0.25 \\
(4.97)\end{array}$ & 0.41 \\
\hline NS501 & $\begin{array}{c}0.00 \\
(4.05)\end{array}$ & $\begin{array}{c}0.00 \\
(4.05)\end{array}$ & $\begin{array}{c}0.00 \\
(4.05)\end{array}$ & $\begin{array}{c}0.13 \\
(4.51)\end{array}$ & $\begin{array}{c}0.38 \\
(5.35)\end{array}$ & $\begin{array}{c}0.75 \\
(6.25)\end{array}$ & $\begin{array}{c}1.13 \\
(7.23)\end{array}$ & $\begin{array}{c}0.69 \\
(6.25)\end{array}$ & $\begin{array}{c}0.63 \\
(6.08)\end{array}$ & $\begin{array}{c}0.38 \\
(5.35)\end{array}$ & $\begin{array}{c}0.13 \\
(4.51)\end{array}$ & 0.38 \\
\hline Romeo & $\begin{array}{c}0.00 \\
(4.05)\end{array}$ & $\begin{array}{c}0.00 \\
(4.05)\end{array}$ & $\begin{array}{c}0.00 \\
(4.05)\end{array}$ & $\begin{array}{c}0.00 \\
(4.05)\end{array}$ & $\begin{array}{c}0.13 \\
(4.51)\end{array}$ & $\begin{array}{c}0.44 \\
(5.55)\end{array}$ & $\begin{array}{c}0.69 \\
(6.23)\end{array}$ & $\begin{array}{c}1.00 \\
(7.01)\end{array}$ & $\begin{array}{c}0.69 \\
(6.25)\end{array}$ & $\begin{array}{c}0.38 \\
(5.35)\end{array}$ & $\begin{array}{c}0.06 \\
(4.28)\end{array}$ & 0.31 \\
\hline Ruby & $\begin{array}{c}0.00 \\
(4.05)\end{array}$ & $\begin{array}{c}0.00 \\
(4.05)\end{array}$ & $\begin{array}{c}0.00 \\
(4.05)\end{array}$ & $\begin{array}{c}0.13 \\
(4.51)\end{array}$ & $\begin{array}{c}0.38 \\
(5.35)\end{array}$ & $\begin{array}{c}0.75 \\
(6.40)\end{array}$ & $\begin{array}{c}1.31 \\
(7.73)\end{array}$ & $\begin{array}{c}1.63 \\
(8.38)\end{array}$ & $\begin{array}{c}0.81 \\
(6.57)\end{array}$ & $\begin{array}{c}0.44 \\
(5.55)\end{array}$ & $\begin{array}{c}0.19 \\
(4.74)\end{array}$ & 0.51 \\
\hline $\begin{array}{c}\text { Roja } \\
\text { (Cherry) }\end{array}$ & $\begin{array}{c}0.00 \\
(4.05)\end{array}$ & $\begin{array}{c}0.00 \\
(4.05)\end{array}$ & $\begin{array}{c}0.00 \\
(4.05)\end{array}$ & $\begin{array}{c}0.06 \\
(4.28)\end{array}$ & $\begin{array}{c}0.31 \\
(5.16)\end{array}$ & $\begin{array}{c}0.94 \\
(6.84)\end{array}$ & $\begin{array}{c}0.94 \\
(6.87)\end{array}$ & $\begin{array}{c}0.63 \\
(6.08)\end{array}$ & $\begin{array}{c}0.31 \\
(5.16)\end{array}$ & $\begin{array}{c}0.19 \\
(4.74)\end{array}$ & $\begin{array}{c}0.00 \\
(4.05)\end{array}$ & 0.31 \\
\hline $\begin{array}{c}\text { BSS908 } \\
\text { (Priya) }\end{array}$ & $\begin{array}{c}0.00 \\
(4.05)\end{array}$ & $\begin{array}{c}0.00 \\
(4.05)\end{array}$ & $\begin{array}{c}0.00 \\
(4.05)\end{array}$ & $\begin{array}{c}0.25 \\
(4.97)\end{array}$ & $\begin{array}{c}0.63 \\
(6.08)\end{array}$ & $\begin{array}{c}1.38 \\
(7.87)\end{array}$ & $\begin{array}{c}1.25 \\
(7.59)\end{array}$ & $\begin{array}{c}0.88 \\
(6.73)\end{array}$ & $\begin{array}{c}0.56 \\
(5.91)\end{array}$ & $\begin{array}{l}0.31 \\
(5.16)\end{array}$ & $\begin{array}{c}0.00 \\
(4.05)\end{array}$ & 0.48 \\
\hline S.Em \pm & 0.00 & 0.00 & 0.00 & 0.17 & 0.17 & 0.37 & 0.29 & 0.20 & 0.14 & 0.18 & 0.16 & \\
\hline $\mathrm{CD}(=0.05)$ & 0.00 & 0.00 & 0.00 & 0.50 & 0.51 & 1.12 & 0.87 & 0.61 & 0.42 & 0.54 & 0.49 & \\
\hline $\mathrm{CV}=$ & 0.00 & 0.00 & 0.00 & 8.27 & 7.19 & 12.82 & 8.99 & 6.55 & 5.25 & 7.53 & 8.12 & \\
\hline
\end{tabular}

Figure in parenthesis signifies the transformation of original value

Fig.1 Weekly average record of different meteorological parameters throughout the entire period of study -2013

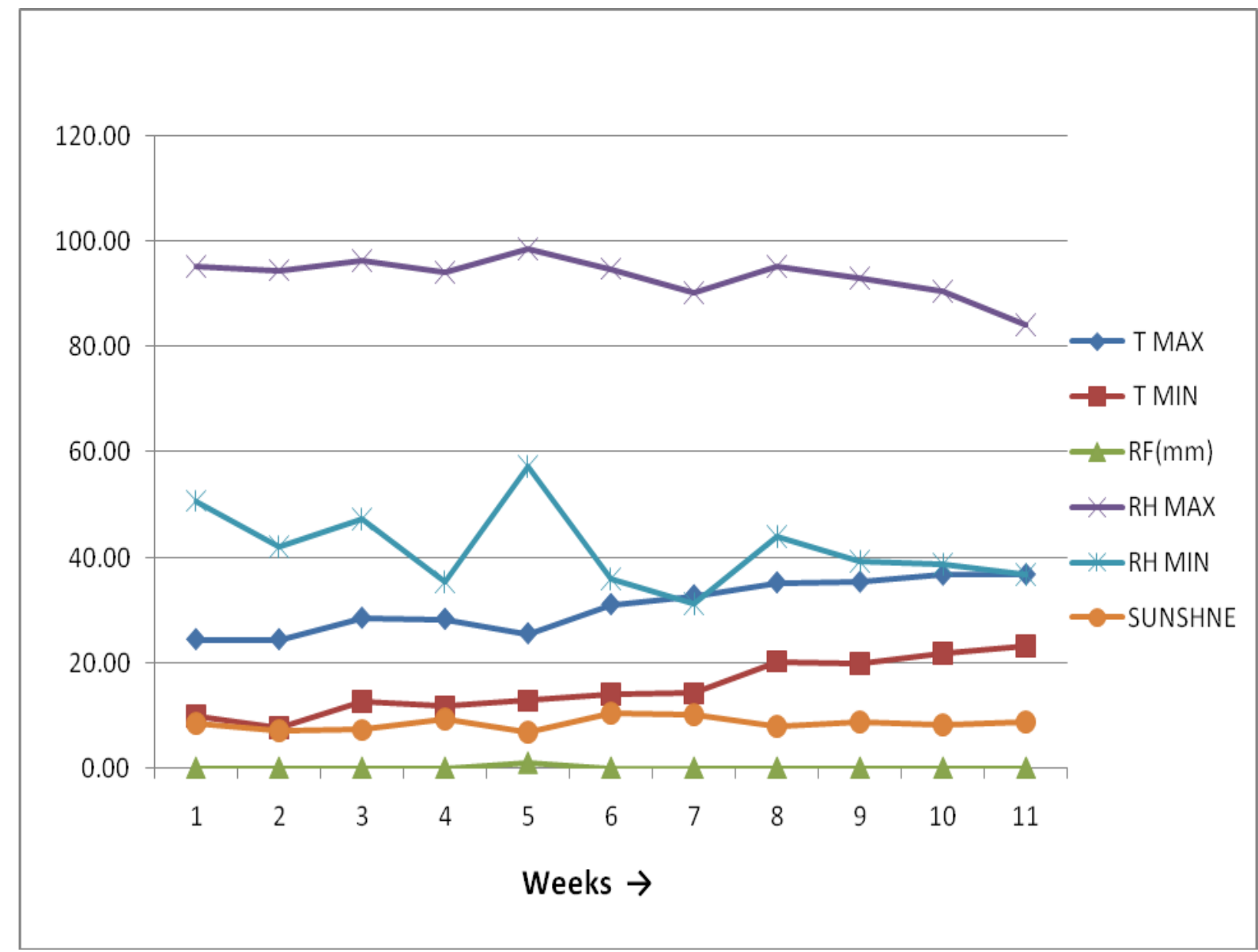

Source: Dept. of Agril. Meteorology \& Physics, B.C.K.V, Mohanpur, Nadia 


\section{Tomato germplasms and natural enemies}

In present experiment it was observed that the tomato ecosystem supports a considerable number and population of natural enemy species. Several species of spiders, predatory coccinellids and one hover fly species were recorded during the experiment. The maximum $(\mathrm{r}=0.500)$ and minimum temperatures $(\mathrm{r}=0.344)$ and sunshine hour $(\mathrm{r}=0.507)$ showed positive correlation with population development of the natural enemy complex whereas maximum $(\mathrm{r}=-0.028)$ and minimum relative humidity $(\mathrm{r}=-0.395)$ showed negative correlation with them. The mean number of the natural enemies varied from 0.31 to 0.51 per plant.

Spiders, coccinellids and hover flies were counted altogether irrespective of the stages. The highest natural enemy population was recorded in Ruby (0.51) followed by BSS 908 Priya (0.48) (Table 5). Whereas lower population was found on Romeo (0.31) and Roja cherry (0.31). In terms of preference of natural enemy, the tomato germplasms can be arranged in the following sequence (higher to lower): Ruby> BSS 908 (Priya) $>$ Patherkuchi $>$ NS 501 $>$ Romeo $=$ Roja.

Insects and plants are in an inseparable and continuous process of co-evolution system. Harmful insects are suppressed by either other insects or microbes or toxic mechanisms or by plant defense mechanisms. These ensure sustainability of crop yield and as well as a balance or homeostasis of the system.

Always emphasis has been given on increase production to feed ever growing human population. Modern-day-production system integrates improved seed or plant materials, new fertilizer regimes and mostly synthetic pesticide-based chemical protection technology to ensure a higher quantum of yield. Insect-plant-interaction is a dynamic relationship. Whenever a plant species is modified to evade or suppress pest attack, it triggers a set of change in the candidate pest and in turn, its immediate next-generation is likely to evolve with a newer mechanism that will help the species to overcome plantdefence barrier and feed on the plant. The special defence attribute of the genotype becomes redundant. For this reason, it is always very difficult to obtain a truly resistant crop cultivar or genotype. However, as long as plants remain, insect or insects will feed on them and from this complex frame, we have to extract better yields to feed the growing population.

The present-day-protection concept emphasizes on the holistic approach which considers the pests, the crop, the environment and the natural enemies altogether. The cost factor also remains under the purview. In brief, this is the system approach in plant protection. This approach ensures better protection and at the same time, maintains the homeostasis at the best possible level. Among the different options available, host plant resistance is one of the effective tools for reducing insect damage. Plant characteristics have been recognized as important factors for resistance reaction. Resistance mechanism in plants against insects may be due to one or more of Preference/non-preference, Antibiosis and Tolerance.

Same kind of observation was also reported by different researcher, Chaudhuri et al., (2001) found that the whitefly population reached highest level during middle of February and high level was maintained from mid-February to mid-March. Hildebrand et al., (1993) and Raghava (2010) reported that antibiosis was the main mechanism of exhibiting tolerance to insect pests. Leite et al., (1999) on the other hand opined that relative tolerance/resistance of tomato genotypes comes from a combination 
different resistance mechanisms, i.e., morphological characters as well as chemical and physiological character of the genotypes. Gajendra et al., (1998) reported that Pusa Early Dwarf, ArkaVikas and PusaGaurva showed tolerance through preference-non preference mechanism. Ashfaq et al., (2012) reported that hair length and hair density on lower leaf surface, as well as thickness of leaf lamina significantly correlated with the infestation. Many researchers reported that wild tomato accessions such as Lycopersicon pimpinellifolium (A-1921) and Lycopersicon hirsutum f. sp. glabratum (B-6013) as resistant sources for ToLCV (Banerjee and Kalloo, 1989 and Banerjee and Kalloo, 1990).

The susceptible genotype of the present experiment (NS 501) certainly does not have any of the resistance mechanisms. Hence, this is simply out of consideration. The best option is definitely the tolerant/resistant types but incidentally, in the present study, none was found. The most promising one in the present study was Patherkuchi. Genotypes Ruby, Romeo, Roja cherry, Priya were moderately susceptible and further studies can be initiated to explore their potentiality. The present study was confined to screening of some potential germplasms for resistance/tolerance and mechanism of resistance was not explored. It is implied that relative tolerance showed by an entry (Patherkuchi) was exerted by any or combination of the three standard resistance mechanisms, that is, preference-non preference, antibiosis and tolerance.

\section{References}

Acquaah, G. (2002). Horticulture: Principles and Practices. New Jersey, Prentice Hall.

Alam, T., Tanweer, G., Goyal, G.K. (2007). Stewart Postharvest Review, Packaging and storage of tomato puree and paste. Res. Art., 3(5):1-8.

Anonymous. (2011). Product Profiles of Tomato. National Horticulture Board. http://agriexchange.apeda.gov.in/links.a spx. (accessed on 15 July 2013).

Anonymous. (2012). National Horticulture Production Database, MoA, GoI.

Ashfaq, M., Sajjad, M., Ane, N.M. and Rana, N. (2012). Morphological and chemical characteristics of tomato foliage as mechanisms of resistance to Helicoverpaarmigera (Hübner) (Lepidoptera: Noctuidae) larvae. African Journal of Biotechnology, 11 (30): 7744-775.

Banerjee, M.K. and Kalloo, G. (1989). The inheritance of earliness and fruit weight in crosses between cultivated tomatoes and two wild species of Lycopersicon. Plant Breeding, 102: 148- 152.

Banerjee, M.K. and Kalloo, G. (1990). Nature of resistance to tomato leaf curl virus (TLCV) in two species of Lycopersicon. Haryana Agricultural University Journal of Research, 20: 225- 228.

Chaudhuri, N., Deb, D.C. and Senapati, S.K. (2001). Biology and fluctuation of white fly (Bemisiatabaci Genn.) population on tomato as influenced by abiotic factors under Terai Region of West Bengal. Indian J. Agric. Res., 35 (3): 155 - 160.

Freedman, N.D., Park, Y., Subar, A.F., Hollenbeck, A.R., Leitzmann, M.F., Schatzkin, A. and Abnet, C.C. (2008). Fruit and vegetable intake and head and neck cancer risk in a large United States prospective cohort study. International Journal of Cancer, 122 (10): 23302336. doi:10.1002/ijc.23319. PMID 180 92323. (accessed on 17 June 2013).

Gajendra, C., Ganguli, R.N., Kaushik, U.K., Dubey, V.K., Chandrakar, G., Reddy, P.P., Kumar, N.K.K. and Verghese, A. (1998).Resistance to the fruit borer, Helicoverpaarmigera in tomato. In: 
Advances in IPM for horticultural crops. Proceeding of the First National Symposium on Pest Management in Horticultural Crops: environmental implications and thrust, Bangalore, India, pp. 73-74.

Hildebrand, D.F., Brown, G.C., Jackson, D.M., Hamilton, K.T.R. (1993). Effects of some leaf emitted volatile compounds on aphid population increase. Journal of Chemical Ecology, 19 (9): 1875-1887.

Leite, G.L.D., Picanco, M., Lucia, T.M.C.D. and Moreira, M.D. (1999). Role of canopy height in the resistance of Lycopersicon hirsutum f. glabratum to Tutaabsoluta (Lep., Gelechiidae). Journal of Applied Entomology, 123 (8): 459-463.

Musuna, A.C.Z. (1983). A potentially furious pest Whitefly, Zimbabwe agric. J., 8 (4): 143-146.

Naika, S., Van, L.J.J., De Goffau, M., Hilmi, M. and Van Dam, B. (2005). Cultivation of tomato: Production, processing and marketing. Agromisa Foundation and CTA, Wageningen, pp92.

Reddy, D.S. (2010). Applied Entomology. New Vishal Publications, p. 218-231.
Shidfar, F., Froghifar, N., Vafa, M., Rajab, A., Hosseini, S., Shidfar, S. and Gohari, M. (2011). The Effects of Tomato Consumption on Serum Glucose, Apolipoprotein B, Apolipoprotein A-I, Homocysteine and Blood Pressure in Type 2 Diabetic Patients. International Journal of Food Sciences and Nutrition, 62 (3): 289-294. PMID21138408. (accessed 12 May 2013).

Shukla, V. and Prabhakar, B.S. (1987). Effect of plant density on yield and losses caused by fungus and insects in brinjal.Agricultural Sci. Digest. India, 7 (3): $148-150$.

Singh, K. (2014). Evaluation of tomato genotypes and its reaction against ToLCV causing leaf curl disease in tomato (Solanum lycopersicon L.). Journal of Experimental Biology and Agricultural Sciences, 2(1S):120-125.

Zhang, C.X., Ho, S.C., Chen, Y.M., Fu, J.H., Cheng, S.Z. and Lin, F.Y. (2009). Greater vegetable and fruit intake is associated with a lower risk of breast cancer among Chinese women. International Journal of Cancer, 125 (1):181-188. doi: 10.1002/ ijc. 24358. P M I D19358284. (accessed on 18 July 2013).

\section{How to cite this article:}

Prahlad Sarkar, Satyajit Hembram and Siddikul Islam. 2018. Host Plant Preference of Sucking Pest to Different Tomato Genotypes under West Bengal Conditions. Int.J.Curr.Microbiol.App.Sci. 7(11): 3244-3252. doi: https://doi.org/10.20546/ijcmas.2018.711.374 